\title{
Extração de Características de Perfil e de Contexto em Redes Sociais para Recomendação de Recursos Educacionais
}

\author{
Crystiam Kelle Pereira ${ }^{1}$, Fernanda Campos' ${ }^{1}$, Victor Ströele', José Maria N. David ${ }^{1}$, \\ Regina Braga ${ }^{1}$ \\ ${ }^{1}$ Departamento de Ciência da Computação - Universidade Federal de Juiz de Fora - \\ Juiz de Fora - MG - Brasil
crystiamkelle@gmail.com, fernanda.campos@ufjf.edu.br, victor.stroeledice.ufjf.br, jose.david@ufjf.edu.br, regina.braga@ufjf.edu.br

\begin{abstract}
This paper presents a proposal that seeks to generate individualized educational recommendations, using information generated spontaneously in Social Networks. The goal is to identify characteristic of user's profile and context through the use of these systems and his/her social interaction and then make consistent educational recommendations.
\end{abstract}

Resumo. Este trabalho apresenta uma proposta que busca gerar recomendações educacionais individualizadas, usando informações geradas espontaneamente nas Redes Sociais. O objetivo é identificar características do perfil e do contexto do usuário através do uso desses sistemas e da sua interação social e então, fazer recomendações educacionais aderentes ao seu perfil e contexto.

\section{Introdução}

Nos últimos anos nota-se um aumento na busca por informações através da Internet. Essa busca tem ocorrido tanto através de cursos formais, nas modalidades à distância e semipresenciais, quanto na procura informal por conteúdos de interesse. Existem inúmeros recursos educacionais distribuídos em diferentes repositórios, que abordam um conjunto amplo de assuntos e que possuem objetivos educacionais distintos. Em virtude disso, a escolha adequada desses recursos é um desafio para os educadores e para os próprios alunos.

Nesse cenário, um sistema de recomendação (SR) tem um papel importante para auxiliar educadores e alunos a encontrarem recursos educacionais relevantes e pertinentes aos seus perfis e ao contexto em que estão inseridos. Segundo Burke (2002), um SR pode ser "qualquer sistema que produza recomendações individualizadas ou que tenha o efeito de guiar o usuário de forma personalizada para objetos do seu interesse ou que lhes sejam úteis dentre diversas opções possíveis".

Para que se torne possível gerar recomendações personalizadas, observa-se a necessidade de informações que ajudem a definir o perfil do usuário e auxiliem na identificação de suas necessidades e interesses. Por outro lado, observam-se alguns sistemas que capturam, espontaneamente, uma grande quantidade de dados dos usuários, bem como a interação entre eles, evidenciando, muitas vezes, suas preferências 
e interesses. Esse é o caso, por exemplo, das Redes Sociais. Segundo Junco (2011), o site de rede social mais popular entre estudantes universitários é o Facebook ${ }^{l}$. De acordo com uma pesquisa realizada pela Quacquarelli Symonds (Vanozzi; Bridgestock, 2013) entre 2012 e 2013, com 918 entrevistados em 26 países distribuídos pela Europa, Ásia, América Latina, América do Norte e África, o uso do Facebook entre os estudantes varia entre 78 e $96 \%$ nessas regiões, sendo que a América Latina é a região que mais utiliza o Facebook.

Nesse sentido, essa pesquisa busca extrair e explorar as informações disponíveis nas redes sociais, bem como o interesse dos seus usuários em estarem atualizados através do seu uso. Por meio da extração de informações disponíveis nas redes sociais, busca-se identificar e inferir características, preferências e interesses educacionais dos usuários, usando técnicas de Extração de Texto e Web Semântica. O objetivo principal é a identificação de características do perfil e do contexto do usuário a partir de informações geradas, espontaneamente, através do uso das redes sociais, para auxiliar no processo de seleção e recomendação de recursos educacionais adequados a esse perfil. Além disso, busca-se explorar o longo tempo dispendido pelos estudantes em redes sociais, levando até eles orientações e recursos educacionais.

Esse trabalho pretende ainda contribuir com o projeto BROAD (Busca e Recuperação de Objetos de Aprendizagem a Distância) (Campos et al., 2012) que engloba pesquisas relacionadas a investigação e adoção de tecnologias de software, tais como ontologias, serviços web semânticos, agentes e workflow para construir uma arquitetura para a busca personalizada por objetos de aprendizagem, bem como para a sua composição em conteúdos educacionais. Este trabalho pretende evoluir a proposta do projeto BROAD, acrescentando características de perfil e do contexto educacional dos usuários, através da aquisição automática de informações disponibilizadas por usuários de redes sociais, possibilitando assim, a recomendação de recursos educacionais personalizados.

O artigo está organizado da seguinte forma: na seção 2 são apresentados alguns trabalhos relacionados, na seção 3 é dada uma visão geral da arquitetura de recomendação, nomeada BROAD-RSI, já na seção 4 são apresentados a proposta, o protótipo desenvolvido, baseado na extração de alguns itens de perfil do Facebook, e uma avaliação do modelo de recomendação. Por fim, na seção 5 são feitas as considerações finais.

\section{Trabalhos relacionados}

Em Fritzen et al. (2012) é apresentada uma proposta que busca modelar o contexto a partir de mensagens de redes sociais, e usar esse contexto para melhorar a relevância na recuperação de documentos na Web, tendo como objetivo apoiar a aprendizagem colaborativa. Dwivedi e Bharadwaj (2012) apresentam uma proposta de recomendação baseada na avaliação de conteúdo em uma rede social educacional, que foi criada no escopo do trabalho apresentado por eles. Os alunos compartilham uns com os outros as suas classificações para recursos didáticos, sendo considerados o estilo de aprendizagem e o nível de conhecimento para atribuir pesos diferentes às recomendações. Fernandes e

\footnotetext{
${ }^{1}$ www.facebook.com
} 
Siqueira (2013) apresentam uma abordagem para melhorar o entendimento e conhecimento dos interesses e preferências dos usuários a partir de informações disponíveis em sistemas colaborativos, mais especificamente redes sociais. A proposta é extrair automaticamente um perfil do usuário, sendo para isso consideradas as postagens dos usuários nas redes sociais. Esse trabalho não tem como objetivo principal a recomendação, no entanto menciona essa possibilidade.

Casagrande et al. (2013) apresentam uma técnica de recomendação baseada na construção implícita de perfis de usuários através da observação dos valores de metadados dos conteúdos que esses usuários acessam. A técnica de recomendação proposta utiliza a filtragem colaborativa combinada com uma técnica de agrupamento de usuários por interesses similares. Em Ferreira et al. (2013) é apresentado o UbiGroup, um modelo de recomendação ubíqua de conteúdo educacional para grupos de alunos. Esse modelo leva em conta o perfil dos alunos e o contexto onde eles estão inseridos. $\mathrm{O}$ UbiGroup faz uso de agentes de software para realizar o processo de recomendação. Feita a associação são realizados filtros considerando o perfil e o contexto do aluno, que são definidos manualmente.

O modelo de recomendação, abordado neste artigo, se difere das propostas apresentadas em dois sentidos: primeiro, na forma como são definidos os itens de perfil e o contexto do usuário, usando dados gerados, espontaneamente, através da interação em Redes Sociais; segundo, no seu objetivo final que é gerar recomendações especificamente educacionais e individualizadas, sendo necessário para isso usar diferentes técnicas para inferência e filtragem de elementos educacionais dentre as diversas informações disponíveis nos sistemas citados. Vale citar ainda a criação de um mapeamento semântico entre as informações de perfil do usuário e os metadados de recursos educacionais catalogados.

\section{Arquitetura de Recomendação do BROAD-RSI}

A arquitetura do BROAD-RSI (Sistema de Recomendação Baseado nas Interações Sociais) (figura 1) pretende atingir os seguintes objetivos: (1) especificar uma estratégia para extrair informações acerca do interesse educacional e informações básicas de perfil dos usuários, usando para isso a rede social Facebook; (2) determinar itens de perfil educacional do usuário a partir das informações extraídas; (3) enriquecer o perfil extraído, buscando tópicos de interesses implícitos; (4) gerar a representação semântica desse perfil e (5) considerar as informações extraídas para sugerir recursos educacionais apropriados.

A arquitetura do BROAD-RSI pode ser dividida em 5 módulos: Extração de Informação; Definição de Perfil Educacional; Enriquecimento do Perfil; Representação Semântica e Sistema de Recomendação. O processo de recomendação pode ser visto na figura 2.

\subsection{Extração de Informações}

O processo de aquisição de informações do perfil inicia-se a partir do login do usuário pela sua conta do Facebook. Após realizar o login, o usuário deve aceitar as permissões necessárias do aplicativo para que as informações sejam extraídas. 


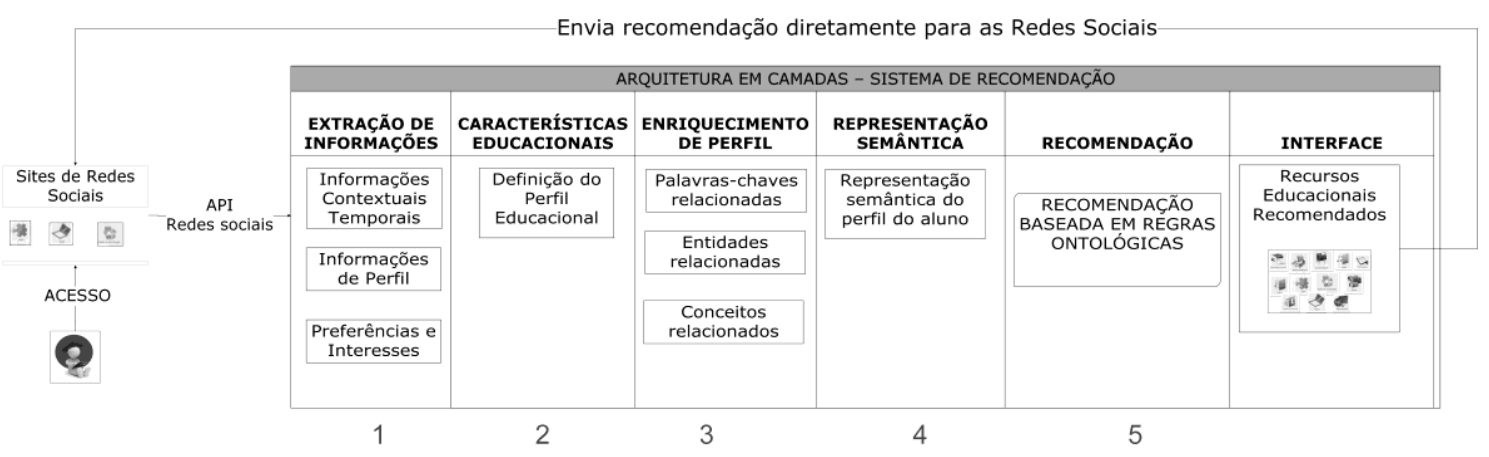

Figura 1 - Arquitetura do BROAD-RSI

Através de sua API (Application Programming Interface), o Facebook disponibiliza informações referentes às conexões de cada usuário, informações públicas do seu perfil (nome, idade, cidade de nascimento e residência, dados educacionais, dados profissionais, idiomas falados), seus interesses e preferências (livros, músicas, filmes, jogos, páginas, entre outros) e ainda os grupos dos quais ele participa. No contexto deste trabalho, são extraídas as informações básicas do perfil, o horário de cada postagem, os idiomas falados ou compreendidos, os interesses e preferências, que são capturados a partir de grupos, páginas e itens curtidos e seguidos.

\subsection{Definição do Perfil Educacional}

Depois de extraídas as informações citadas anteriormente, faz-se necessário filtrá-las, buscando quais, dentre os diversos interesses do usuário, podem ser consideradas de cunho Educacional. Para isso são consideradas: as áreas de interesse definidas no Perfil Educacional do usuário e as páginas curtidas, cuja categoria esteja no âmbito Educacional, tais como, páginas referentes a faculdades, escolas, linhas de pesquisa, entre outras. É extraído também o nível educacional do usuário (Ensino Médio, Graduação, Pós-Graduação). Apesar das informações educacionais não serem obrigatórias, elas estão presentes no perfil de muitos usuários. Segundo um estudo feito no escopo deste trabalho com uma amostra de 251 usuários, 92 deles possuíam, em seu perfil, informações sobre áreas educacionais de interesse, ou seja, 36,65\%. Considerando o amplo uso do Facebook, o quantitativo de pessoas beneficiadas por uma recomendação educacional seria considerável.

Além das informações relacionadas aos interesses educacionais dos usuários, são capturadas também aquelas relativas às suas preferências em relação aos dias, horários e períodos (manhã, tarde e noite) de acesso à rede social. Pretende-se, com isso, enviar recomendações educacionais nos horários mais oportunos de acordo com o perfil de acesso do usuário. São extraídas, ainda, as preferências por tipos diferentes de Mídias, a partir dos quantitativos dos diferentes tipos de recursos compartilhados pelo usuário no Facebook, tais como Livros, Músicas, Vídeos, Imagens e Jogos. Essa preferência ajuda a priorizar recursos de acordo com o seu formato, não sendo usada, portanto, para eliminar uma recomendação, mas apenas para dar mais importância e prioridade a um subconjunto delas. 


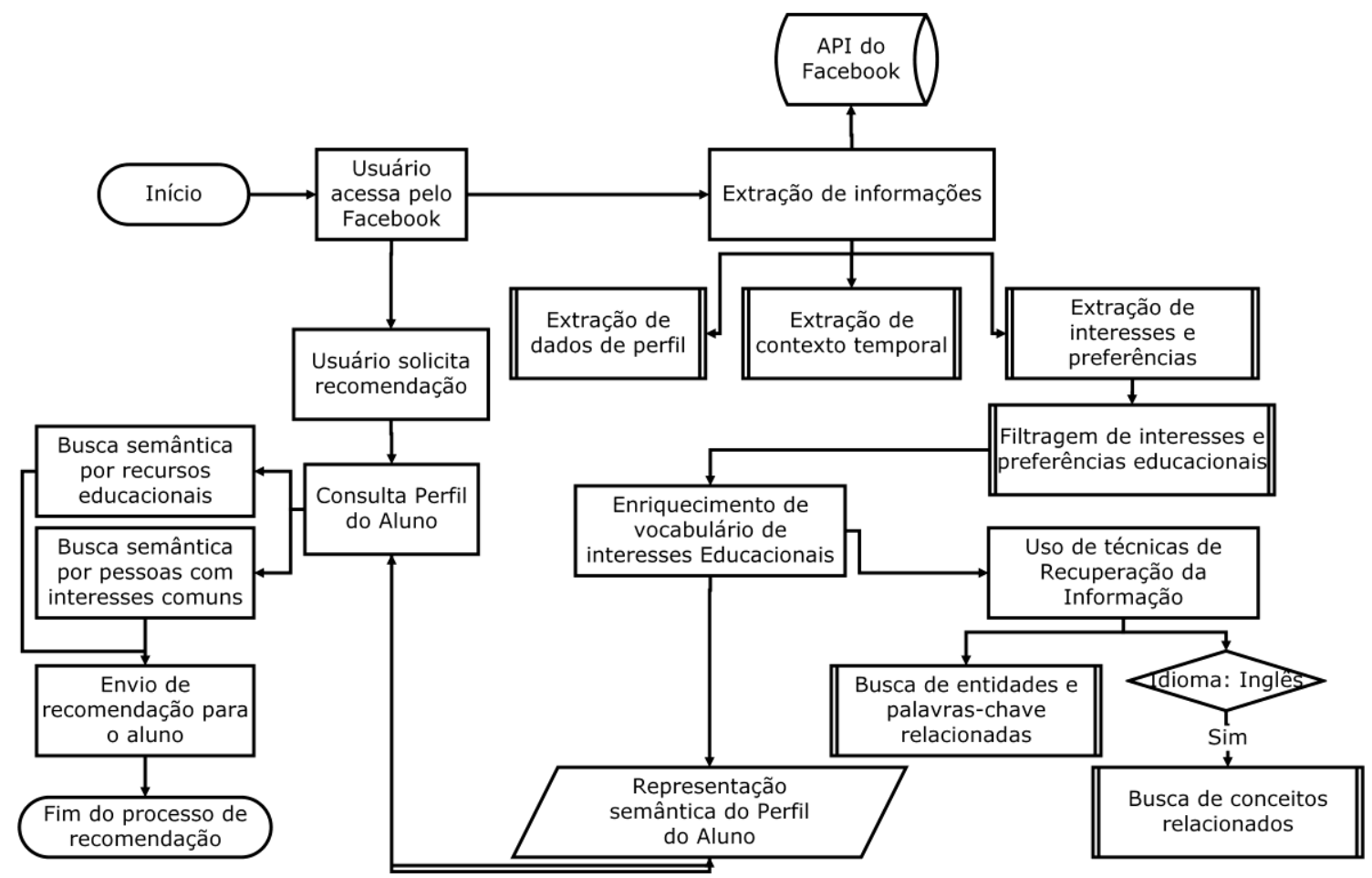

Figura 2 - Fluxo de Recomendação

\subsection{Enriquecimento de Perfil}

Durante a extração das informações educacionais, observou-se que apenas o nome ou título de uma área de interesse, item ou página eram insuficientes para compreender os tópicos de interesse de um usuário e gerar uma recomendação de forma ampla que envolva, por exemplo, interesses correlatos. Sendo assim, houve a necessidade de enriquecimento do Perfil Educacional extraído.

O Enriquecimento dá-se com maior ênfase nos tópicos de interesse e para isso é usado o texto descritivo de cada uma das páginas e de cada área de interesse. A partir do texto descritivo são identificadas as entidades e os conceitos associados a um determinado tema. Para a identificação de entidades e conceitos, é usada a API AlchemyAPI8. Segundo Orchestr8 (2011), essa API baseia-se na aplicação de processamento estatístico de linguagem natural e de algoritmos de aprendizagem de máquina para a análise do conteúdo e extração de metadados semânticos, fornecendo assim, um conjunto de ferramentas para análise de conteúdo e anotações. Dentre as ferramentas oferecidas pela API, são utilizadas neste trabalho: extração de entidades, palavras-chave e conceitos e detecção do idioma do texto. A utilização desses recursos permite buscar termos e conceitos implícitos no texto, enriquecendo o perfil do usuário.

\subsection{Representação Semântica}

A representação semântica é feita através de uma ontologia que envolve classes referentes a tópicos de interesse, instituições onde a pessoa estudou ou trabalhou, grau de formação educacional, idiomas dominados pelo usuário, preferências por mídias e horário do acesso, conforme mostrado na figura 3. 


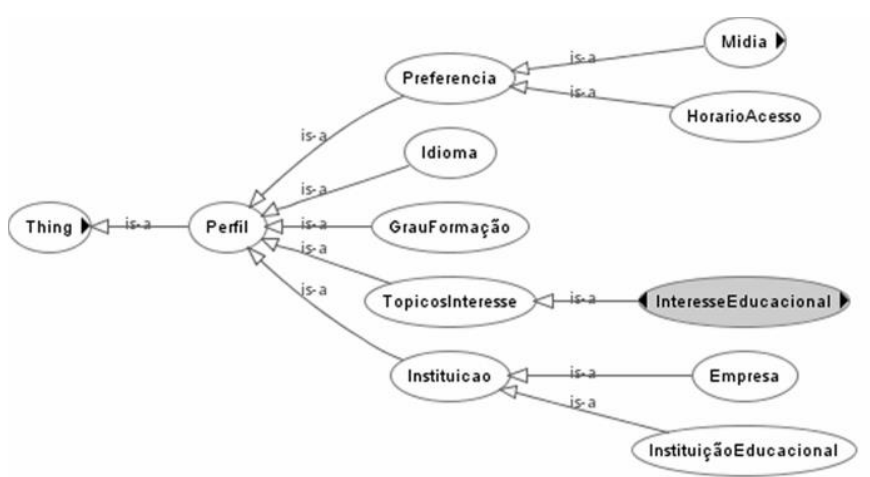

Figura 3 - Ontologia do Perfil do Usuário

Os interesses educacionais, bem como a extensão dos conceitos associados a eles, também dão origem a uma base semântica de interesses educacionais extraídos do Facebook, exemplificada na figura 4. Essa representação semântica é gerada automaticamente como consequência do uso do protótipo desenvolvido no escopo deste trabalho. A classe representa uma área de interesse e suas subclasses representam os conceitos, entidades e palavras-chave, resultantes do enriquecimento do perfil.

Para a representação semântica dos recursos educacionais foi usada a ontologia criada para o padrão OBAA (Viccari et al, 2010), que engloba também os padrões LOM (IEEE, 2002) e IMS AccessForAll (Behr et al., 2012).

\subsection{Recomendações de Recursos Educacionais e de Pessoas}

A recomendação dos recursos educacionais é feita pelo BROAD-RSI a partir da relação estabelecida entre o perfil do usuário e os metadados do recurso educacional. Geralmente os recursos educacionais são catalogados usando padrões de metadados, como por exemplo: IEEE LOM e OBAA, permitindo assim a busca e reutilização desses recursos. Não se pretende, neste trabalho, investigar ou atuar sobre a catalogação e representação do contexto dos recursos. O projeto BROAD já prevê a catalogação de recursos educacionais utilizando um subconjunto de metadados do LOM e considerando algumas categorias do padrão OBAA, incluindo ainda uma ontologia para a representação desse conjunto de metadados (Campos et al, 2012).

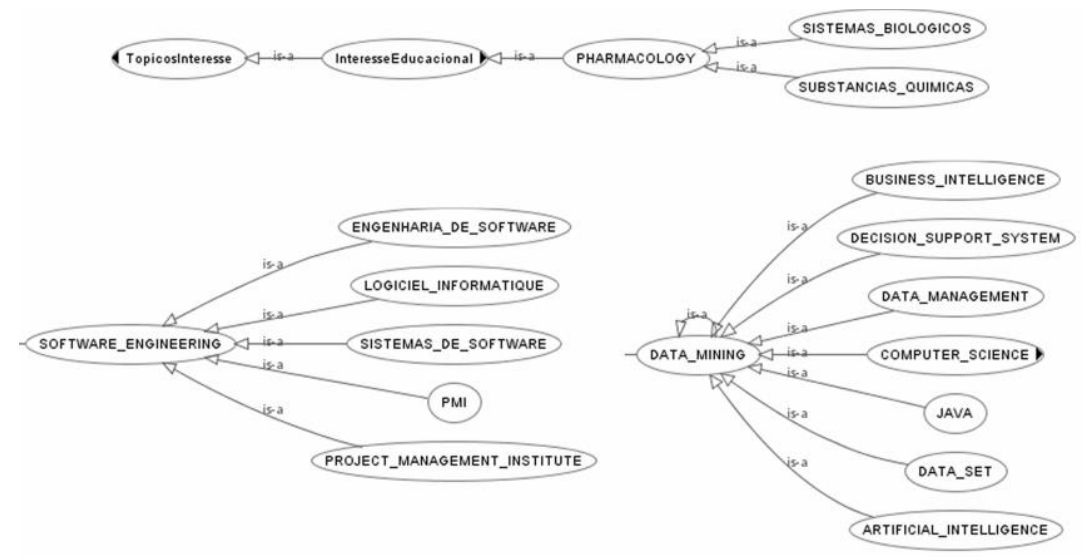

Figura 4 - Ontologia de Áreas de Interesses Educacionais 
A etapa de recomendação tem foco em estabelecer regras que façam a busca e os filtros adequados, considerando as informações extraídas das redes sociais. Na tabela 1 são apresentados os metadados considerados, bem como a relação estabelecida entre eles e as informações extraídas.

Tabela 1 - Relação entre metadados e informações extraídas

\begin{tabular}{|l|l|}
\hline Metadados LOM & Informações extraídas \\
\hline General.Title & Área de interesse \\
\hline General.Language & Idiomas falados \\
\hline General.Keyword & Área de interesse \\
\hline Technical.Format & Preferência por mídias (Visuais/Verbais) \\
\hline Educational.Context & Grau de Formação \\
\hline Educational.TypicalAgeRange & Idade \\
\hline
\end{tabular}

A recomendação de pessoas é feita após a identificação das áreas de interesse do usuário. Tendo as áreas identificadas, é possível extrair redes de interesses e indicar pessoas que tenham interesses comuns a um determinado usuário. A formação das redes de interesse propicia um ambiente de discussão dos temas de interesse, onde as contribuições dos seus membros enriqueçam o conhecimento de todos que fazem parte dela. Mais que a própria discussão que pode ser gerada dentro das redes de interesse, é possível explorar a recomendação de recursos educacionais pela técnica de filtragem colaborativa (FC) (Burke, 2002).

\section{Metodologia de Avaliação}

Para permitir a avaliação do Sistema de Recomendação de Recursos Educacionais a partir da extração de informações do Facebook foi implementado um Protótipo. Através desse protótipo é possível o usuário fazer login, usando a sua conta do Facebook, dar permissão de uso às suas informações e, então, são extraídas as informações já citadas anteriormente. Para o desenvolvimento do protótipo, foi utilizada a linguagem de programação Java e ainda as seguintes tecnologias: Tomcat, Jena, Sparql, API Graph através da biblioteca RestFB, AlchemyAPI e MySQL.

Os experimentos para avaliação da proposta consistem na utilização de validação por cenários. Segundo Ferreira (2013), essa abordagem vem sendo utilizada pela comunidade científica para avaliação de ambientes sensíveis ao contexto, como é o caso da nossa proposta.

Considere a seguinte situação: alguns professores de áreas distintas fizeram o cadastro de recursos educacionais no repositório BROAD, utilizando alguns metadados do padrão LOM. Os professores cadastraram diversos materiais e preencheram alguns metadados, sendo que, aqueles considerados neste trabalho foram registrados conforme mostra a tabela 2. Para o preenchimento do metadado General.Keyword foi oferecido aos professores várias opções que já fazem parte de uma base semântica de áreas de interesse, base essa alimentada a partir das áreas de interesse encontradas nos perfis do Facebook daqueles usuários que utilizam o protótipo.

Considere ainda três cenários: Cenário 1, Cenário 2 e Cenário 3, onde três usuários com perfis distintos estarão acessando o Protótipo, são eles, respectivamente, o Usuário1, Usuário2 e Usuário3, cujos dados extraídos do Facebook estão resumidos na tabela 3 . 
Tabela 2 - Cenários: catalogação de recursos educacionais

\begin{tabular}{|c|c|c|c|c|}
\hline \multirow{2}{*}{$\begin{array}{l}\text { Metadados } \\
\text { padrão LOM }\end{array}$} & \multicolumn{4}{|c|}{ Descrição dos Recursos Educacionais } \\
\hline & $\begin{array}{c}\text { Recurso } \\
\text { Educacional } 1\end{array}$ & $\begin{array}{c}\text { Recurso } \\
\text { Educacional } 2\end{array}$ & $\begin{array}{c}\text { Recurso } \\
\text { Educacional } 3\end{array}$ & $\begin{array}{c}\text { Recurso } \\
\text { Educacional } 4 \\
\end{array}$ \\
\hline Title & $\begin{array}{l}\text { Análise por Pontos } \\
\text { de Função }\end{array}$ & $\begin{array}{l}\text { Apostila } \text { de } \\
\text { Mineração de dados }\end{array}$ & $\begin{array}{l}\text { Software } \quad \text { Engineering. A } \\
\text { Practitioners Approach }\end{array}$ & $\begin{array}{lr}\text { Farmacologia: } & \text { A } \\
\text { ciência } & \text { da } \\
\text { Farmacologia } & \\
\end{array}$ \\
\hline Language & Espanhol & Português & Inglês & Português \\
\hline Keyword & $\begin{array}{l}\text { Engenharia_de_soft } \\
\text { ware } \\
\text { Project_managemen } \\
\mathrm{t} \\
\text { Software_engineerin } \\
\mathrm{g}\end{array}$ & $\begin{array}{l}\text { Banco_de_dados } \\
\text { Database_manage } \\
\text { ment_system } \\
\text { Data_management } \\
\text { Data_mining }\end{array}$ & $\begin{array}{l}\text { Engenharia_de_software } \\
\text { Information_systems } \\
\text { Information_technology } \\
\text { Project_management } \\
\text { Software_engineering }\end{array}$ & $\begin{array}{l}\text { Pharmacology } \\
\text { Sistemas_biologic } \\
\text { os } \\
\text { Substancias_quim } \\
\text { icas }\end{array}$ \\
\hline Format & Vídeo & Livro & Livro & Vídeo \\
\hline Context & Pós-Graduação & Pós-Graduação & Graduação & Graduação \\
\hline TypicalAge & 18- & $18-$ & $15-18 / 18-$ & $15-18 / 18-$ \\
\hline
\end{tabular}

Tabela 3 - Cenário dos usuários

\begin{tabular}{|l|l|l|l|}
\hline Tipo de Informação & Perfil do usuário & \\
\hline & Cenário 1: Usuário1 & Cenário2: Usuário2 & Cenário3:Usuário3 \\
\hline Área de interesse Educacional & PHARMACOLOGY & $\begin{array}{l}\text { ENGENHARIA_DE_SOFTWARE } \\
\text { PROJECT_MANAGEMENT }\end{array}$ & DATA_MINING \\
& 20 & 23 & 40 \\
\hline Idade & Graduação & Graduação & Pós-Graduação \\
\hline Grau de escolaridade & Vídeo & Livro & Vídeo \\
\hline Preferências Mídias & Português/Espanhol & Português/Espanhol & Português/Inglês \\
\hline Idiomas falados &
\end{tabular}

Os usuários Usuário1, Usuário2 e Usuário3 estão precisando de alguns recursos educacionais para ajudá-los a recordar conceitos a respeito da sua área de interesse e, então, decidiram utilizar o protótipo do BROAD-RSI. Ao fazerem o login, utilizando suas contas do Facebook, o sistema faz a extração dos dados de perfil dos usuários e considera os dados citados na tabela 1 para recomendação. O texto descritivo de cada área de interesse educacional, disponível no Facebook, é submetido a métodos disponíveis na APIAlchemy e dá origem a um novo conjunto de termos (entidades, conceitos e palavras-chaves) associados a cada uma das áreas. Tanto a área explicitamente declarada no perfil do Facebook, quanto os termos implícitos são associados ao perfil do usuário e, então, é gerada a representação semântica desse perfil. Além disso, as áreas de interesse declaradas e implícitas são acrescentadas à base semântica de áreas de interesse, conforme mostrado na figura 4.

Feito isso, o sistema apresenta aos usuários uma tela para a escolha de uma área de interesse, dentre as diferentes áreas declaradas e implícitas no perfil do usuário. Feita a escolha, o sistema retorna ao usuário um conjunto de recursos educacionais correspondentes ao seu interesse e, ainda, uma lista de usuários que possuem interesses comuns. Essa lista é adquirida a partir de uma busca semântica na base de usuários do BROAD-RSI. É feito também um cálculo de similaridade entre esses usuários, de tal forma que quanto mais interesses comuns os usuários possuem, mais similares eles são.

Feito o processo descrito, os usuários Usuário1, Usuário2 e Usuário3 recebem as recomendações mostradas respectivamente no Cenário 1, Cenário 2 e Cenário 3 , conforme figura 5 , onde pode-se observar que foram consideradas para a recomendação: as áreas de interesse, o idioma e o grau de escolaridade. $\mathrm{O}$ tipo de recurso educacional não impede a recomendação, apenas prioriza de acordo com a preferência do usuário. 
Após os usuários visualizarem os recursos educacionais sugeridos, eles têm a possibilidade de fazer uma avaliação das recomendações.

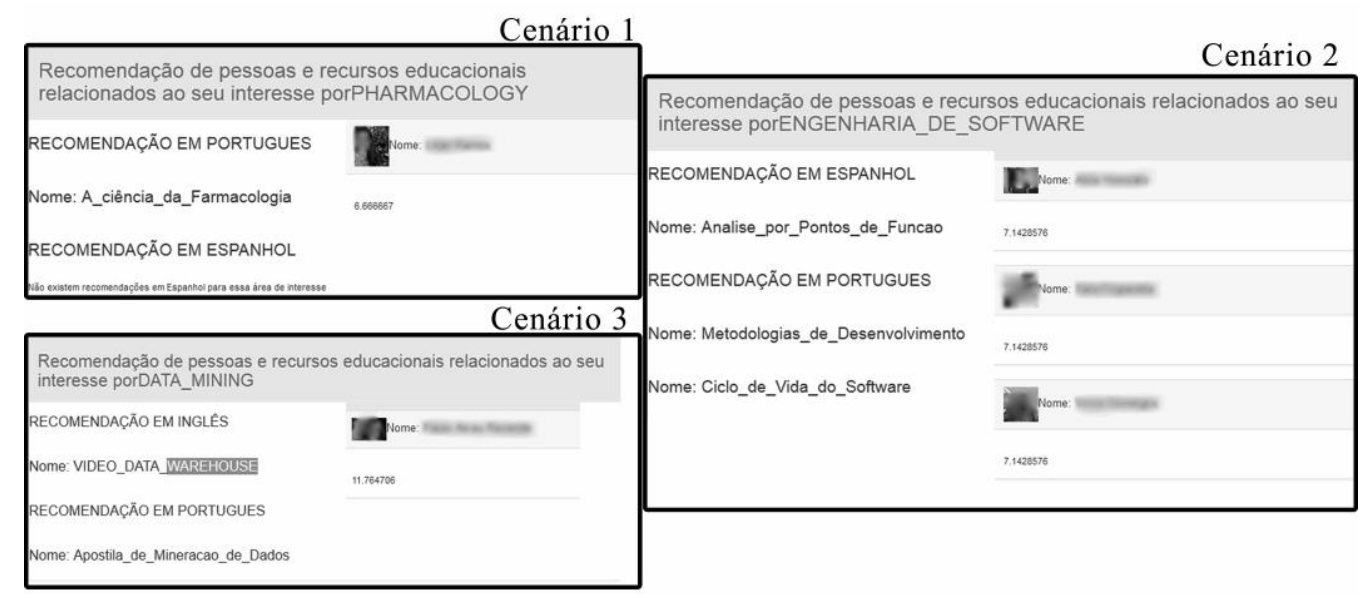

Figura 5 - Recomendações distintas específicas ao perfil do usuário

\section{Considerações Finais}

O presente trabalho apresentou uma proposta de identificação automática de características do perfil educacional do usuário, utilizando informações extraídas da Rede Social Facebook, incluindo também uma proposta de Recomendação de recursos educacionais que consideram esse perfil.

A arquitetura proposta pode ser resumida em cinco camadas, sendo elas: (1) extração das informações, (2) definição do perfil Educacional, (3) enriquecimento do perfil, (4) representação semântica e (5) recomendação. Para a extração de informações são consideradas informações disponíveis no site de Rede Social Facebook. Através dessas informações são definidos dados básicos de perfil do usuário e suas preferências educacionais. Após a extração foram usadas técnicas de análise textual e web semântica para estender o vocabulário relativo aos interesses educacionais, trazendo novos interesses implícitos. Uma representação semântica do perfil do usuário é criada e através de associações entre ela e os metadados dos recursos educacionais são selecionados um conjunto de recursos apropriados a cada usuário.

O sistema de recomendação apresentado neste trabalho traz aspectos inovadores no sentido de extrair e considerar informações geradas espontaneamente nas redes sociais, mais especificamente no Facebook, gerando recomendações individuais, especificamente educacionais, que estejam coerentes com os interesses e as preferências identificados a partir da análise das informações oriundas desse sistema. Outra contribuição é a possibilidade da recomendação de pessoas, possibilitando a formação de uma rede de interesses em torno de um determinado tema.

A pesquisa no sentido de criar um ambiente educacional de recomendação usando informações extraídas de redes sociais ainda está em andamento e terá como passos futuros: (1) a ampliação de informações extraídas do Sistema Facebook; (2) a consideração da rede de interesses para melhorar a qualidade das recomendações; (3) o aprimoramento da técnica de recomendação usada pelo BROAD-RSI; (4) a inclusão da recomendação dentro das redes sociais; (5) realização de uma avaliação quantitativa e qualitativa a respeito da eficácia e qualidade das recomendações feitas. 


\section{Agradecimentos}

Esta pesquisa é parcialmente apoiada pela UFJF, FAPEMIG, CAPES e CNPq.

\section{Referências}

Behr A., Primo T., Viccari R. (2012). "An Ontology for the OBAA Metadata Standard". In: Anais do $23^{\circ}$ Simpósio Brasileiro de Informática na Educação (SBIE 2012), Rio de Janeiro-RJ, Novembro de 2012

Burke, R. (2002) "Hybrid recommender systems: survey and experiments". User Modeling and User- Adapted Interaction, 12, 4, 331-370, 2002.

Campos, F. et al. (2012) "BROAD Project: Semantic Search and Application of Learning Objects". IEEE Technology and Engineering Education (ITEE).

Casagrande M. F. R., Kozima G., Wilirich R. (2013). “Técnica de Recomendação Baseada em Metadados para Repositórios Digitais Voltados ao Ensino". In: Anais do $24^{\circ}$ Simpósio Brasileiro de Informática na Educação (SBIE 2013), Campinas-SP, p. 677-686 de Novembro de 2013

Dwivedi P. and Bharadwaj K.K. (2012) "E-learning recommender system for learners in online social networks through association retrieval", In: Proceedings of the CUBE International Information Technology Conference, 6(1), p. 676-681.

Ferreira L. G. A., Barbosa J. L. V., Gluz J. C. (2013). "Um modelo de Recomendação Ubíqua de Conteúdo para Grupos de Aprendizes”. In: Anais do $2^{\circ}$ Congresso Brasileiro de Informática na Educação (CBIE 2013), Campinas, 2013, p. 679 - 706.

Fernandes G. T., Siqueira S. W. M. (2013) "Building Users Profiles Dynamically Through Analysis of Messages". In: Collaborative Systems In International Conference WWW/Internet (ICWI 2013), Forth Worth, Texas, USA; 2013

Fritzen, E.; Siqueira, S. W. M. and Andrade, L. C. V. (2012) "Recuperação Contextual de Informação na Web para Apoiar Aprendizagem Colaborativa em Redes Sociais". In: Simpósio Brasileiro de Informática na Educação, 2012, Rio de Janeiro.

IEEE Learning Technology Standard Committee (LTSC). (2002) Standard for Learning Object Metadata (LOM), 2002.

Junco, R. (2011) "The relationship between frequency of Facebook use, participation in Facebook activities, and student engagement”. Computers \& Education, 58, 162-71.

Orchestr8. Alchemyapi. http://www.alchemyapi.com/, 2011. Acessado em 03/07/2014.

Vanozzi, M. and Bridgestock, L. (2013) 'Students' online usage: Global Market trends report. QS TopUniversities.com 2013". Disponível em: http://www.topuniversities.com/sites/qs.topuni/files/Students-Online-Useage-GlobalTrends-Report-2013-nc.pdf

Viccari, R.; Gluz, j. and Passerino, L. M., et al. (2010) "The OBAA Proposal for Learning Objects Supported by Agents”. In: MASEIE Workshop - AAMAS 2010, Toronto, Canada. 